\title{
Star formation history: secular processes in "main sequence" galaxies versus merger-driven starbursts
}

\section{Matthieu Bethermin}

CEA Saclay France, email: matthieu.bethermin@cea.fr

\begin{abstract}
Some recent works indicate that most star-forming galaxies follow a main sequence in the SFR-stellar mass plane with a surprisingly low scatter of $\approx 0.2 \mathrm{dex}$, suggesting that the star formation in these objects is driven by secular processes. Nevertheless, Herschel identified a population of starbursting galaxies, probably triggered by mergers, which display a large excess of specific star formation rate ( $\mathrm{SSFR}=\mathrm{SFR} / \mathrm{Mstar})$ compared to the main sequence. We will present a new set of models for the contribution of these two populations to the IR/sub-mm luminosity function, but also to source counts selected at various wavelengths.

Our model is based on the stellar mass function of star-forming galaxies, the distribution of sSFR measured at $z=2$ and its double-Gaussian decomposition, and the observed evolution of the main sequence in the sSFR-Mass plane as a function of redshift. We found that the non-Schechter bright-end of the LF is due to the starbursting galaxies, which represent only $4 \%$ in number density and $15 \%$ in luminosity density. This fraction of starbursts is remarkably constant with the redshift at $0<z<2$, contrary to naive expectation from hierarchical merging. It thus suggests that the majority of stars in the Universe were formed through secular processes. We will then discuss the contribution of starbursting and main sequence galaxies to the number counts and the selection effects towards starbursts sources for various flux-limited IR/sub-mm samples.

We will also present studies of the clustering properties of the main sequence and starburst galaxies at $z 2$. These measurements suggest strong links between star formation rate, stellar mass and halo mass in the main sequence galaxies. In addition, we will present some clues suggesting that main sequence and starbursting galaxies follows the same $\mathrm{M}^{*}$-Mhalo relation."
\end{abstract}

